Journal of World-Historical Information

David Eltis and Paul Lachance

\title{
Comments on Manning, Zhang, and Yi's "Volume and Direction of the Atlantic Slave Trade, 1650-1870"
}

Patrick Manning, Yun Zhang and Bowen Yi’s essay is, to our knowledge, the first since Slavevoyages went live in late 2008 to address the question of the size and direction of the largest coerced migration in global history. Given that the Markov Chain Monte Carlo (MCMC) methodology that they employ has moved to center stage of statistical inquiry in this eight-year interval, their work is indeed timely. Our commentary has three parts. First we point out several problems in their use of data from the Trans-Atlantic Slave Trade Database (hereafter TASTDB) on which their estimates are based; second we call attention to our own estimates of the volume of the slave trade which are higher than those mentioned in the essay; and third we argue that, as currently constituted, their work is broadly supportive of the statistical picture that we drew in 2008 - albeit without the benefit of MCMC.

The TASTDB, accessible on the website Slavevoyages.org, is very much a collaborative work in progress. Its goal is to record information on all transatlantic slaving voyages whose organization is documented in extant sources. The expanded SPSS version contains 279 variables that permit systematic statistical analysis of the transoceanic movement of captives. ${ }^{1}$ These include 198 data variables, 3 technical variables, and 78 imputed variables. $^{2}$ Data variables comprise information coded directly from primary sources. Technical variables include a distinct identification number assigned to each voyage, and groupings of voyages by nationality, rig, route,

Volume 2-3, No. 2 (2014-15) | ISSN 2169-0812 (online)

DOI 10.5195/jwhi.2015.36 | http://jwhi.pitt.edu 
and period of time used in imputing the number of captives embarked and/or disembarked when such information is not documented in sources. Imputed variables have the same values as corresponding data variables when these are available, but assign imputed values when documented information is not available. Such imputed values are inferred from other data variables for the same voyage or from patterns of data variables for other voyages with similar characteristics. In summary, imputed variables provide an estimate of the total number of slaves transported by documented voyages, including cases where the number is not reported in the documentation.

For the Manning et al. analysis, the four key variables are tslavesd, a data variable showing total slaves leaving Africa; slaximp, the imputed variable for slaves leaving Africa that adds to tslavesd imputed numbers for slave departures where raw data for a voyage are missing; slaarriv, a data variable for slaves disembarking from a voyage; and slamimp, the imputed variable that, like slaximp for the other side of the Atlantic, adds missing numbers where raw data are unavailable. But most importantly we took one further step in developing our estimates. In a separate interface on the website (slavevoyages.org/assessment/estimates), we took into account not just voyages where no slave numbers had survived, but made an additional allowance for those voyages for which documentation has not yet been found (and, for some, never will be found). This final step allowed us to provide our own assessment of the overall volume and direction of the slave trade, including voyages of which there is no record. ${ }^{3}$

The essay by Manning et al. has three flaws. First, data from the TASTDB may have been incorrectly entered. How is it possible to generate negative values for losses at sea and the larger number of arrivals than departures in most decades from the 1670s to the end of the slave trade in Figure 5 on p. 143 - results that are clearly impossible? Raw data for the number of voyages, embarkations, and arrivals by decade in rows 16 to 23 of Appendix 5 remain at odds with the TASTDB. The only row that matches figures from the database is for voyages with disembarked slaves, but it matches the number of voyages for which tslavesd (embarkations) are coded, not slaarriv (arrivals). In short, it is possible that mistakes in data entry distort the results obtained by application of new statistical procedures to the raw data. Since the results are similar to our own estimates, we wonder how correct entry of the two data variables - tslavesd and slaarriv - would affect the comparison. ${ }^{4}$

Second, Manning et al. also misreport the results from imputed variables in the TASTDB. Embarkations from the 1650s to the 1860s in the final row of Table 2c, supposedly based on slaximp, are stated to be 8,045,576 as opposed to the correct figure of 9,787,772. ${ }^{5}$ The Voyages totals for the same time periods are only 7 and 5 percent lower than the estimated totals generated by the paper's "multi-method" and MCMC approaches respectively rather than 20 to 25 percent less as claimed by the authors in the abstract, at the bottom of p. 144, and in Table 2.

Third, except for a brief dismissive reference at the bottom of p. 131, Manning et al. ignore our own carefully constructed and separate "Estimates" interface located under "Assessing the Slave Trade" in the top banner of the Voyages website. It is imperative to recognize that there are two quite distinct imputation process-*ses executed with different databases and displayed with separate user interfaces with their own pages in the website. The first-creates imputed values in the main database, which for the most part supply data for missing values for voyages that have been documented as having been organized. These are the estimates that Manning et al. attempt to engage with. Our second estimating procedure not only has its own interface, but that interface is supported by a separate essay, a multipage spreadsheet and an SPSS dataset that generates the data used by the Voyages estimates interface. ${ }^{6}$ This set 
of estimates and the supporting documentation is not discussed in their paper. Far from being "recently published" (p. 131) as Manning et al. claim, these have been available since 2008.

The gist of our procedures is that we estimate, period by period, how complete the records of different national carriers are. The default table on our "Estimates" page shows the results that Manning et al. do not engage with. As countless references across the literature have made clear, our 2008 estimates raise total embarkations to 12.5 million. This entirely calls into question the authors' conclusion at the top of p.146 that their results point to "a consistent downward bias" in the "Slave Voyages method of estimation." Far from the Manning et al. estimates being $25 \%$ higher, as they claim, they are in fact, at 12.2 million, slightly lower than ours.

It would be more accurate to conclude that, in its currently flawed condition, the MCMC method of reassessing our data broadly supports our findings of eight years ago. The MCMC method assumes that no historical knowledge of the unknown voyages exists, and in terms of specific voyages this is indeed correct. But anyone who reads our 2010 estimates-method.pdf will quickly see that there is a range of contemporary documentary contextual evidence that shapes our findings on the incidence and size of gaps in the data. The knowledge base for the missing voyages is not zero.

Finally, at the end of their essay Manning et al. ask two questions: "First, can one create a more detailed regional breakdown on both the African and American sides of the Atlantic? Second, could one estimate the movement of captives from specific African regions to specific regions in the Americas-thus, from the Bight of Biafra to Virginia or from Senegambia to the French Antille?” (p. 146). In fact, such breakdowns by individual year have been available at www.slavevoyages.org/assessment/estimates since 2008 for every transatlantic pairing of regions that the slave trade created.

Anyone can derive estimated figures different from, but perhaps just as valid as, our own by projecting from the hard data that we have made available on Slavevoyages. The MCMC methodology may be one way of doing this, but until Manning et al. correct their errors and engage fully with our own contribution to this important topic, we cannot know. In addition even when corrected it seems most unlikely that their procedures will be able to generate estimated annual breakdowns departures and arrivals for every region involved in the slave trade on both sides of the Atlantic.

\section{NOTES}

${ }^{1}$ Downloadable from the website: http://www.slavevoyages.org/voyage/download/tastdb-exp-2010.sav.

2 The difference between imputed and data variables, and the methods used in imputing values when not directly recorded in documents, are explained in detail in the essay " Construction of the Trans-Atlantic Slave Trade Database: Sources and Methods" in the section "Understanding the Database" under "Voyages Database" (http://www.slavevoyages.org/voyage/understanding-db/methodology-4). The variables are fully described in the

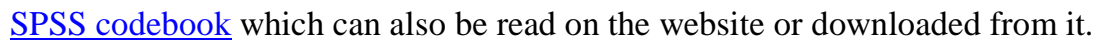

${ }^{3}$ We do, of course, anticipate that researchers will continue to discover previously undocumented voyages and additional information about voyages already in the database. The website contains a Contribute section to facilitate incorporation of this new information as it is submitted. The 2010 version of the database was updated in December 
2015, to include such new information, increasing the size of the database from 34,948 to 35,938 voyages. For the sake of comparability, however, we will use data and estimates from 2010 in our comments here.

${ }^{4}$ To assist them in this, as well as to give readers access to our corrections, we provide a revised Appendix 5, available at http://www.slavevoyages.org/voyage/download (see the Estimates section, file = ManningAppx5rev_2016122.xlsx) where the correct data display in red font alongside the erroneous data in black font.

${ }^{5}$ See the correct totals for slaximp and slamimp added to rows 56 and 58 of ManningAppx5rev_2016218. Slaximp is estimated for 31,822 voyages, including 6,455 for which region of embarkation in Africa is unknown and could not be imputed.

${ }^{6}$ All three of these files are available on the downloads page at http://www.slavevoyages.org/voyage/download (see the Estimates paragraph).

\section{(cc) EY Articles in this journal are licensed under a Creative Commons Attribution 4.0 United} States License.

\section{ULIS D-Sunt}

This journal is published by the University Library System of the University of Pittsburgh as part of its D-Scribe Digital Publishing Program and is cosponsored by the University of Pittsburgh Press. 